\section{RSP}

http://www.rsp.fsp.usp.br/
Revista de Saúde Pública

\title{
Tendência de mortalidade por câncer de boca e faringe no Brasil no período 2002-2013
}

Lillia Magali Estrada Perea', Marco Aurélio Peres", Antonio Fernando Boing'"', José Leopoldo Ferreira Antunes ${ }^{\mathrm{IV}}$

I Universidade Federal de Santa Catarina. Programa de Pós-Graduação em Saúde Coletiva. Florianópolis, SC, Brasil " University of Adelaide. Adelaide Dental School. Australian Research Center for Population Oral Health. Adelaide, Australia

III Universidade Federal de Santa Catarina. Departamento de Saúde Pública. Florianópolis, SC, Brasil

iv Universidade de São Paulo. Faculdade de Saúde Pública. Departamento de Epidemiologia. São Paulo, SP, Brasil

\section{RESUMO}

OBJETIVO: Analisar a tendência das taxas de mortalidade por câncer de boca e de faringe no período de 2002-2013 no Brasil segundo sexo, sítio anatômico e macrorregião do país.

MÉTODOS: Os dados sobre mortalidade foram obtidos junto ao Sistema de Informações sobre Mortalidade e os dados das populações foram obtidos junto ao Instituto Brasileiro de Geografia e Estatística. A tendência das taxas padronizadas por sexo e faixa etária foi calculada por regressão de Prais-Winstein, obtendo a sua variação percentual anual e os respectivos intervalos de confiança de 95\%, analisados segundo sexo, macrorregião e sítio anatômico.

RESULTADOS: O coeficiente médio de mortalidade por câncer de boca foi de 1,87/100.000 habitantes e permaneceu estável durante o período estudado. O coeficiente de mortalidade por câncer de faringe foi de 2,04/100.000 habitantes e apresentou variação percentual anual de -2,6\%. Aproximadamente oito em cada 10 óbitos ocorreram entre homens. Observou-se aumento nas taxas por câncer de boca na região Nordeste (variação percentual anual de 6,9\%) e diminuição na região Sudeste (variação percentual anual -2,9\%). Mortalidade por câncer de faringe diminuiu nas regiões Sudeste e Sul com variação percentual anual de -4,8\% e -5,1\% respectivamente. Mortalidade por câncer de amígdala, outras glândulas salivares maiores, hipofaringe e outras partes não especificas de boca e faringe, apresentaram tendência de declínio enquanto os demais sítios apresentaram estabilidade.

CONCLUSÕES: A mortalidade por câncer de faringe apresentou diminuição no período 2002-2013. O câncer de boca apresentou aumento só na região Nordeste. Mortalidade por câncer de amígdala, outras glândulas maiores, hipofaringe e outras localizações mal definidas de lábio, cavidade oral e faringe mostraram declínio.

DESCRITORES: Neoplasias Bucais, mortalidade. Neoplasias Faríngeas, mortalidade. Mortalidade, tendências. Brasil. 


\section{INTRODUÇÃO}

O câncer de boca e faringe é considerado um problema de saúde pública mundial ${ }^{1}$. Segundo dados da International Agency for Research on Cancer $^{\mathrm{a}}$ (IARC), em 2015, foram diagnosticados 571.386 casos novos da doença no mundo e foram registradas 316.168 mortes. A estimativa é que esses valores aumentem nos próximos anos, chegando a 350 mil óbitos em 2020, resultando em uma taxa de 4,7 mortes para cada 100.000 habitantes.

A tendência de mortalidade devido a essa doença varia segundo a região. Na Europa, as taxas de mortalidade por câncer de boca e faringe vêm diminuindo desde a década de $1970^{2}$, enquanto na Oceania ${ }^{3} \mathrm{e}$ em diversos países da América Latina, essas taxas vêm aumentando desde a década de $1980^{4}$. O Brasil é o país com as maiores taxas de mortalidade em ambos os sítios na América Latina ${ }^{5}$, apresentando aumento desde a década de $1980^{6}$.

São diversos os fatores que podem influenciar a tendência das taxas de mortalidade, como as mudanças na prevalência de exposição aos principais fatores de risco da doença ${ }^{7,8} \mathrm{e} \mathrm{a}$ disponibilidade e acesso a diagnóstico e tratamento precoce. Importantes variações têm ocorrido nesses fatores no contexto nacional desde as últimas décadas do século XX.

Segundo dados do Observatório da Política Nacional de controle do Tabaco ${ }^{\mathrm{b}}$ (INCA), foi observada queda de $46 \%$ no percentual de fumantes entre 1989 e 2013. Já o consumo de álcool, que vinha mostrando aumento desde 1960, apresenta estagnação desde $2000^{9}$. $\mathrm{O}$ vírus do papiloma humano (HPV) também tem sido associado com a carcinogênese dessa doença, especialmente com câncer de orofaringe e amígdala ${ }^{10}$. Porém, essas mudanças têm o potencial de modificar ou diminuir as taxas só em longo prazo, uma vez que os efeitos dos fatores carcinogênicos são cumulativos e de longa latência.

Outro fator importante que pode influenciar a tendência de mortalidade é o diagnóstico em fases precoces da doença e a oferta de terapia adequada. O diagnóstico nos estágios tardios implica um pior prognóstico e diminuição da taxa de sobrevida ${ }^{11}$. Igualmente, os avanços nas técnicas usadas no tratamento também podem influenciar na qualidade de vida do paciente e evitando possíveis recidivas que finalizem em óbito ${ }^{12}$.

Boing et al. ${ }^{6}$ analisaram as tendências das taxas de mortalidade por câncer de boca e faringe entre 1979-2002 no Brasil e observaram maior redução dos valores em sítios anatômicos mais acessíveis à inspeção clínica, sugerindo uma possível conexão entre a facilidade visual ao exame diagnóstico e menores taxas de mortalidade. Entretanto, de acordo com busca eletrônica realizada nas bases bibliográficas SciELO, PubMed, Lilacs e Scopus, desde esse último estudo, não foram encontrados outros estudos de tendência que permitam analisar a evolução das taxas de mortalidade por câncer de boca e faringe segundo sítio anatômico específico.

a International Agency for Research on Cancer. Globocan 2012: estimated cancer incidence, mortality and pevalence worlwide. Lyon: IARC; 2012 [citado 29 out 2017]. Disponível em: http:// globocan.iarc.fr

${ }^{\mathrm{b}}$ Instituto Nacional de Câncer. Observatório da Política Nacional de Controle do Tabaco. Rio de Janeiro: INCA; s.d. [citado 29 out 2017]. Disponível em: http://www.inca.gov.br/ observatoriotabaco/

'Ministério da Saúde (BR), Departamento de Informática do SUS - DATASUS . Sistema de Informações sobre Mortalidade - SIM. Brasília (DF); s.d. [citado 25 out 2017]. Disponível em: http://datasus.saude.gov.br/ sistemas-e-aplicativos/eventos-v/ sim-sistema-de-informacoes-demortalidade
O Brasil se encontra em um processo de transição demográfica e epidemiológica que vem afetando de forma direta a incidência e mortalidade das doenças crônicas não transmissíveis ${ }^{1}$. Esse cenário demanda estudos, seguimentos e análises dessas doenças, entre elas, o câncer de boca e faringe.

O objetivo deste estudo foi analisar as tendências de mortalidade por câncer de boca e faringe no Brasil segundo sítio anatômico, sexo e macrorregião do país durante o período de 2002-2013.

\section{MÉTODOS}

Para analisar a tendência temporal da mortalidade por câncer de boca e faringe no Brasil, foi realizado um estudo ecológico empregando-se os dados dos óbitos ocorridos no país durante o período 2002-2013. Os dados de mortalidade anuais foram obtidos junto ao Sistema de Informações sobre Mortalidade (SIM)c disponibilizados por meio do portal do Sistema de Informática do Sistema Único de Saúde (Datasus). O número de habitantes é 
disponibilizado pelo Instituto Brasileiro de Geografia e Estatística (IBGE)d e originário do Censo do ano 2010 e de estimativas intercensitárias para os demais anos. Os óbitos com sexo e idade ignorados foram excluídos da presente análise e totalizaram 0,05\% dos casos.

Foram analisados os óbitos por câncer de boca (C00.0-C08.9) e de faringe (C09-C14.8), conforme a $10^{a}$ Classificação Internacional de Doenças (CID-10), segundo macrorregião de residência (Norte, Nordeste, Sudeste, Sul e Centro-Oeste), sexo e sítio anatômico. Também foi analisada a tendência temporal das taxas de mortalidade para cada sítio anatômico, que foram agrupados segundo características comuns relativas à localização e histologia do tecido. Esse procedimento garantiu número suficiente de casos e propiciou maior estabilidade às analises.

As taxas de mortalidade por câncer de boca e faringe foram calculadas por 100.000 habitantes e ajustadas pelo método direto por sexo e faixas etárias (com intervalos de cinco anos). Considerou-se como padrão a distribuição porcentual da população mundial fornecida pela Organização Mundial da Saúde ${ }^{13}$ (2001), aplicada ao número total da população mundial de 2015. Esse procedimento foi realizado com o intuito de garantir a comparação dos resultados com estudos anteriores que usam outras populações para padronização.

Para calcular a variação percentual anual (VPA) das taxas, foi usada a regressão de PraisWinsten, que prevê correção de autocorrelação de primeira ordem. A variável dependente foi o logaritmo das taxas, e a variável independente, os anos da série histórica. O cálculo da variação percentual anual das taxas foi realizado com as seguintes fórmulas, como sugerido por Antunes e Waldman ${ }^{14}$.

$$
-1+10^{b}=\Delta
$$

Para o cálculo dos intervalos de confiança:

$$
\Delta_{\text {IC95\% }}=-1+10^{\left(b t t^{*} s e\right)}
$$

Em que " $b$ " corresponde à taxa de crescimento anual. Os valores de " $b$ " e erro padrão (se) foram extraídos da análise de regressão, e o valor de " $t$ " é fornecido pela tabela da distribuição $t$ de Student. A tendência de aumento, declínio ou estagnação foi expressa como VPA, com os respectivos intervalos de confiança (95\%), sendo considerada estacionária a tendência cujo coeficiente de regressão não foi diferente de zero $(\mathrm{p}>0,05)$.

Para facilitar a visualização das tendências, reduzimos o ruído branco nos gráficos das séries históricas, mediante a técnica de médias móveis centrais de ordem 3 . A análise de tendência foi realizada no programa Stata, versão 13.

\section{RESULTADOS}

Entre 2002 e 2013, ocorreram 74.342 óbitos por câncer de boca $(n=35.534)$ e faringe $(n=38.808)$ no Brasil, correspondendo a 3,9\% das mortes por todas as neoplasias no período estudado. Aproximadamente oito em cada 10 óbitos ocorreram entre homens.

Quando analisadas conjuntamente, as taxas de mortalidade por câncer de boca e faringe apresentaram estabilidade tanto nos homens, com coeficiente médio de 6,74/100.000 habitantes, quanto nas mulheres, com coeficiente médio de 1,45/100.000 habitantes. Embora com tendência estável, os homens apresentaram coeficiente médio 4,6 vezes maior do que as mulheres. A relação entre o coeficiente médio masculino e feminino foi de 3,7:1 para o câncer de boca e 6:1 para o câncer de faringe. Essa relação apresentou diminuição de 1,5\% e 1,6\% para mortalidade por câncer de boca e faringe, respectivamente, quando comparadas a

${ }^{\mathrm{d}}$ Instituto Brasileiro de Geografia e Estatística. Censo Demográfico 2010. Rio de Janeiro: IBGE; s.d. [citado 25 out 207]. Disponível em: https://censo2010.ibge.gov. br/resultados.html razão do coeficiente masculino e feminino de 2002 com a de 2013 (dados não apresentados).

Quando analisadas as taxas de mortalidade por câncer de boca e faringe separadamente, foi observada estabilidade na mortalidade por câncer de boca (coeficiente médio de 1,87/100.000 habitantes), e decréscimo na mortalidade por câncer de faringe (coeficiente médio de 2,4/100.000), 
especialmente entre os homens (VPA de -2,7\%). A Figura 1 mostra o comportamento das taxas de mortalidade por câncer de boca e câncer de faringe segundo sexo.

O câncer de boca e o câncer de faringe apresentaram diferenças bem marcadas segundo regiões (Tabela 1). Para o câncer de boca, a região Nordeste apresentou um dos menores coeficientes médios para o período (1,60/100.000), mas com aumento médio anual na mortalidade de 6,9\%. A região Sudeste, por sua vez, apresentou o segundo coeficiente médio mais elevado (2,04/100.000); porém, foi a única região em que houve redução das taxas de mortalidade (VPA de -2,9\%).

Quanto ao câncer de faringe, tanto a região Sudeste quanto a região Sul apresentaram diminuição nas taxas de mortalidade, com VPA de -4,8\% e -5,1\%, respectivamente (Tabela 1). Nas demais regiões, os valores foram estáveis. A série histórica das taxas de mortalidade por câncer de boca e faringe segundo regiões está apresentada na Figura 2.

O sítio anatômico com o maior coeficiente médio no período estudado foi orofaringe $(0,91 / 100.000)$, seguido de outras partes e de partes não específicas da boca $(0,62 / 100.000)$. Foram observadas tendências de diminuição para o câncer de amígdalas (-6,6\%), outras glândulas salivares maiores (-4,9\%), hipofaringe (-5,8\%) e outras localizações não específicas de boca e faringe (-3,8\%). As tendências das taxas de mortalidade nos demais sítios foram estáveis durante o período de estudo. A Tabela 2 descreve o número de óbitos, o coeficiente médio e a VPA das taxas de mortalidade devido ao câncer de boca e faringe segundo sítio anatômico.
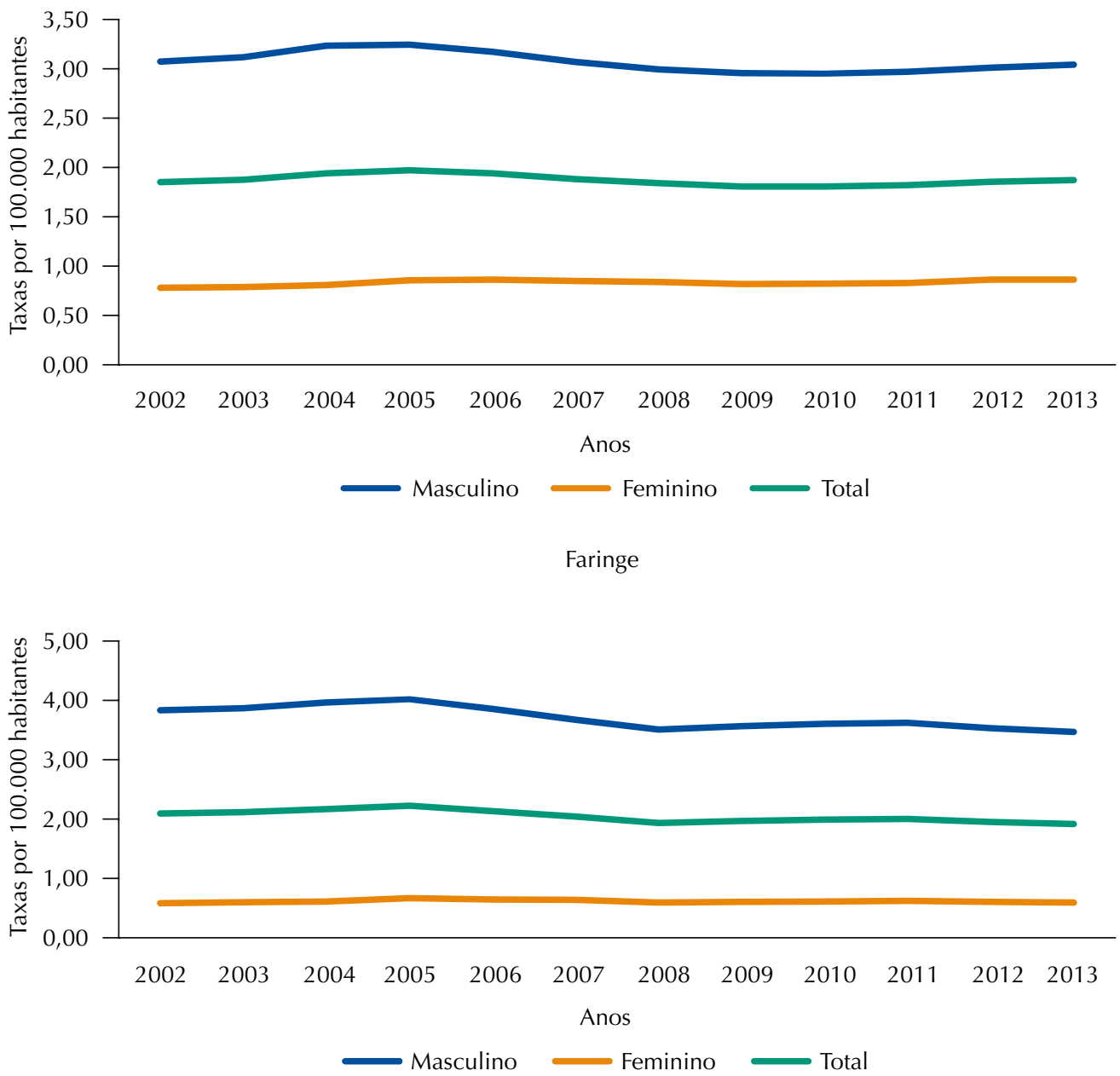

* Taxas padronizadas pela população mundial da $\mathrm{OMS}^{13}$.

Figura 1. Série histórica das taxas* de mortalidade por câncer de boca e faringe segundo sexo. Brasil, 2002-2013. 
Tabela 1. Número e porcentagem de óbitos, coeficiente médio por 100.000 habitantes e tendência das taxas de mortalidade por câncer de boca e faringe segundo regiões. Brasil, 2002-2013.

\begin{tabular}{|c|c|c|c|c|c|c|}
\hline \multirow{2}{*}{ Região } & \multicolumn{2}{|c|}{ Óbitos } & \multirow{2}{*}{$\begin{array}{l}\text { Coeficiente } \\
\text { médio }^{\mathrm{a}}\end{array}$} & \multirow{2}{*}{$\mathrm{VPA}^{\mathrm{b}}$} & \multirow{2}{*}{ IC95\% ${ }^{c}$} & \multirow{2}{*}{ Interpretação } \\
\hline & $\mathbf{n}$ & $\%$ & & & & \\
\hline \multicolumn{7}{|c|}{ Boca } \\
\hline Norte & 1.170 & 3,3 & 1,2 & 5,0 & $-0,9-11,3$ & Estável \\
\hline Nordeste & 8.012 & 22,5 & 1,6 & 6,9 & $1,5-12,6$ & Aumento \\
\hline Sudeste & 18.007 & 50,7 & 2,0 & $-3,0$ & $-4,6--1,4$ & Diminuição \\
\hline Sul & 6.331 & 11,9 & 2,1 & $-2,8$ & $-6,4-0,9$ & Estável \\
\hline Centro-oeste & 2.014 & 5,7 & 1,8 & $-3,6$ & $-8,7-1,8$ & Estável \\
\hline Total & \multicolumn{2}{|c|}{35.534} & 1,9 & $-0,6$ & $-2,6-1,5$ & Estável \\
\hline \multicolumn{7}{|c|}{ Faringe } \\
\hline Norte & 1.185 & 3,1 & 1,1 & 1,9 & $-1,8-5,8$ & Estável \\
\hline Nordeste & 7.549 & 19,4 & 1,5 & 5,9 & $-0,4-12,5$ & Estável \\
\hline Sudeste & 20.021 & 51,6 & 2,3 & $-4,9$ & $-7,9--1,8$ & Diminuição \\
\hline Sul & 7.458 & 19,2 & 2,5 & $-5,1$ & $-7,9--2,3$ & Diminuição \\
\hline Centro-oeste & 2.595 & 6,7 & 2,2 & $-4,9$ & $-9,7-0,3$ & Estável \\
\hline Total & \multicolumn{2}{|c|}{38.808} & 2,0 & $-2,7$ & $-5,0--0,3$ & Diminuição \\
\hline
\end{tabular}

${ }^{\mathrm{a}}$ Taxas padronizadas pela população mundial, $\mathrm{OMS}^{13}$.

Variação percentual anual

c Intervalo de confiança do VPA.

Boca

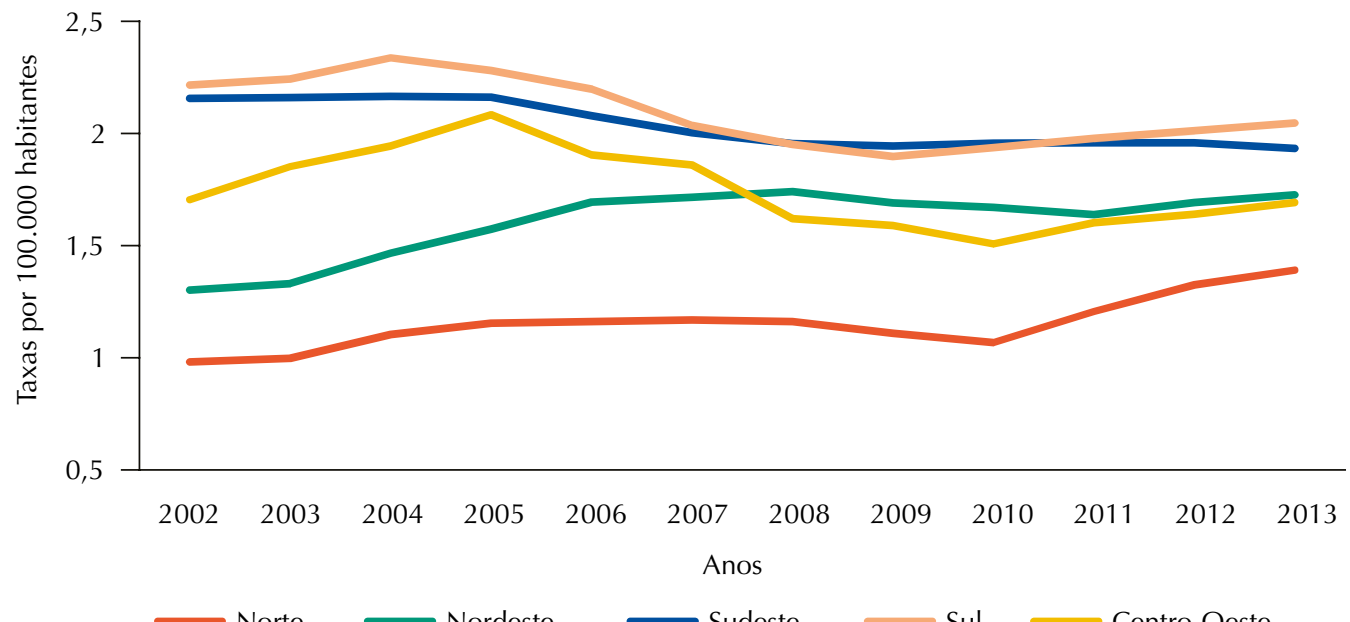

Faringe

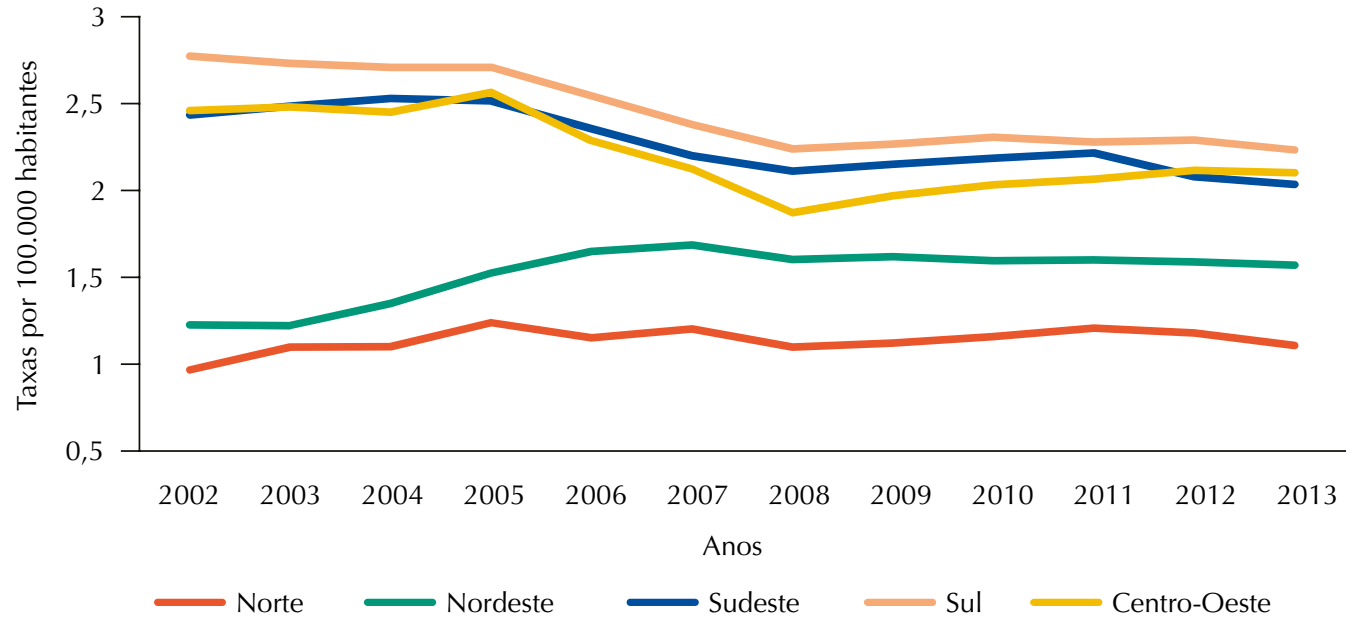

* Taxas padronizadas pela população mundial da $\mathrm{OMS}^{13}$ (2001).

Figura 2. Série histórica das taxas* de mortalidade por câncer de boca e câncer de faringe segundo regiões, Brasil, 2002-2013. 
Tabela 2. Número e porcentagem de óbitos, coeficiente médio por 100.000 habitantes e tendência das taxas de mortalidade por câncer de boca e faringe segundo sítio anatômico. Brasil, 2002-2013.

\begin{tabular}{|c|c|c|c|c|c|c|}
\hline \multirow{2}{*}{ CID-10 } & \multicolumn{2}{|c|}{ Óbitos } & \multirow{2}{*}{$\begin{array}{l}\text { Coeficiente } \\
\text { médio }^{\mathrm{a}}\end{array}$} & \multirow{2}{*}{ VPA $^{b}$} & \multirow{2}{*}{ IC95\% ${ }^{c}$} & \multirow{2}{*}{ Interpretação } \\
\hline & $\mathbf{n}$ & $\%$ & & & & \\
\hline \multicolumn{7}{|c|}{ Sítio anatômico } \\
\hline C00 - Lábio & 589 & 0,8 & $<0,1$ & $-0,1$ & $-7,5-7,8$ & Estável \\
\hline C01 - Base da língua & 4.491 & 6,0 & 0,2 & 2,4 & $-0,9-5,9$ & Estável \\
\hline C02 - Outras partes da língua & 10.326 & 13,9 & 0,5 & $-0,4$ & $-2,5-1,8$ & Estável \\
\hline C03 - Gengiva & 473 & 0,6 & $<0,1$ & $-0,5$ & $-5,1-4,3$ & Estável \\
\hline C04 - Assoalho da boca & 2.040 & 2,7 & 0,1 & 2,7 & $-0,3-5,8$ & Estável \\
\hline C05 - Palato & 2.650 & 3,6 & 0,1 & 0,7 & $-2,2-3,6$ & Estável \\
\hline C06 - Outras partes da boca & 11.683 & 15,7 & 0,6 & $-2,5$ & $-5,3-0,5$ & Estável \\
\hline C07 - Glândula parótida & 2.489 & 3,4 & 0,1 & $-1,6$ & $-6,3-3,3$ & Estável \\
\hline C08 - Outras glândulas & 793 & 1,1 & $<0,1$ & $-5,0$ & $-9,5--0,2$ & Diminuição \\
\hline C09-Amídalas & 1.705 & 2,3 & 0,1 & $-6,7$ & $-9,4--3,9$ & Diminuição \\
\hline C10 - Orofaringe & 17.292 & 0,2 & 0,9 & $-0,7$ & $-3,4-2,0$ & Estável \\
\hline C11 - Nasofaringe & 3.181 & 4,3 & 0,2 & $-0,7$ & $-2,9-1,6$ & Estável \\
\hline C12 - Seio piriforme & 787 & 1,1 & $<0,1$ & $-3,2$ & $-8,4-2,4$ & Estável \\
\hline C13 - Hipofaringe & 4.789 & 6,4 & 0,3 & $-5,9$ & $-9,5--2,1$ & Diminuição \\
\hline C14 - Outras localizações & 11.054 & 14,9 & 0,6 & $-3,8$ & $-6,4--1,2$ & Diminuição \\
\hline \multicolumn{7}{|c|}{ Sítios agrupados } \\
\hline C01 + C02 - Língua & \multicolumn{2}{|c|}{14.817} & 0,8 & 0,4 & $-1,2-2,0$ & Estável \\
\hline $\begin{array}{l}\text { C07 + C08 - Glândula parótida } \\
\text { e outras glândulas maiores }\end{array}$ & \multicolumn{2}{|c|}{3.282} & 0,2 & $-2,8$ & $-6,0-0,4$ & Estável \\
\hline $\begin{array}{l}\text { C09 + C10 - Amídalas e } \\
\text { orofaringe }\end{array}$ & \multicolumn{2}{|c|}{18.997} & 1,0 & $-1,3$ & $-3,6-1,1$ & Estável \\
\hline
\end{tabular}

a Taxas padronizadas pela população mundial, $\mathrm{OMS}^{13}$.

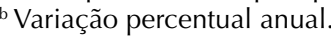

c Intervalo de confiança do VPA.

\section{DISCUSSÃO}

As tendências temporais das taxas de mortalidade por câncer de boca e faringe no Brasil no período de 2002-2013 mostraram diferentes padrões de acordo com sexo, regiões do país e sítios anatômicos da doença. Segundo sexo, embora estáveis, as taxas de mortalidade por câncer de boca e faringe nos homens foram maiores do que nas mulheres durante todo o período de estudo. No entanto, a razão entre os sexos apresentou diminuição ao longo da série histórica investigada, possivelmente relacionada com a aquisição, por parte das mulheres, de hábitos associados ao estilo de vida masculino.

Essa discrepância nas taxas entre sexos é observada tanto internacionalmente ${ }^{14}$ quanto no Brasil ${ }^{15}$ e está possivelmente relacionada com a exposição no passado aos principais fatores de risco e proteção, como o tabaco, cuja prevalência de consumo no Brasil é maior nos homens do que nas mulheres ${ }^{16}$. Outra possível explicação para essa discrepância entre sexos é a frequência de consulta regular ao dentista, que costuma ser maior nas mulheres do que nos homens ${ }^{17}$, e as diferenças socioeconômicas entre gêneros ${ }^{18}$.

$\mathrm{Na}$ análise por sítio anatômico, foi observada diminuição na mortalidade por câncer de amígdala, câncer de outras glândulas salivares maiores, câncer de hipofaringe e por câncer de localizações não específicas de boca e faringe no Brasil. Observou-se estabilidade das tendências das taxas dos demais sítios anatômicos, alguns deles de difícil diagnóstico ou de alta incidência. Orofaringe, por exemplo, considerado anteriormente como sítio anatômico de tendência crescente ${ }^{19}$, apresentou estabilidade no período 2002-2013. Ainda assim, foi o sítio anatômico com o maior coeficiente médio por 100.000 habitantes no período estudado. 
A estagnação ou a estabilidade observada nesses sítios posteriores, que são considerados de difícil inspeção no exame clínico, pode ser consequência de melhorias no acesso aos serviços de saúde e técnicas cirúrgicas mais conservadoras e eficazes ${ }^{20}$.

No presente estudo, observamos diferenças entre macrorregiões. $\mathrm{O}$ aumento observado na tendência da mortalidade por câncer de boca na região Nordeste sugere ter havido melhoria dos sistemas de informação no período estudado, o que poderia gerar maior registro de óbitos para o cálculo ${ }^{21}$. Porém, esse aumento também pode estar relacionado com mudanças de estilos de vida, aumentando a exposição a fatores de risco. A melhoria do sistema de informação pode ter influenciado parcialmente o aumento observado nas taxas; portanto, esse resultado deve ser interpretado com cautela. As demais regiões do país apresentaram estabilidade nas taxas investigadas.

A diferença nas tendências das taxas entre regiões também pode ter sido influenciada pelo acesso a serviços de saúde, uma vez que as regiões Norte e Nordeste têm as menores taxas de profissionais de saúde em atividade e o maior percentual de pessoas que referem nunca ter consultado dentista ${ }^{22}$. A consulta regular com o profissional de saúde, que influi no diagnóstico oportuno de lesões pré-cancerosas, também apresenta grandes variações entre regiões, que podem explicar o comportamento diferenciado das taxas de mortalidade devido ao câncer de boca e faringe em cada uma delas, pois regiões mais ricas têm maior prevalência de consultas ao dentista ${ }^{23}$.

Assim como no estudo sobre as tendências da mortalidade devido ao câncer de boca e faringe no Brasil para o período 1979-2002, a tendência da mortalidade por câncer de boca no Brasil entre 2002 e 2013 apresentou estabilidade para ambos os sexos. No entanto, Boing et al. ${ }^{6}$ observaram aumento na mortalidade por câncer de faringe, enquanto, no período 2002-2013, essa taxa apresentou diminuição. Igualmente, a mortalidade por câncer de orofaringe, hipofaringe e outros sítios não definidos mostraram aumento no período 1979-2002, enquanto foi observada estabilidade para esses sítios anatômicos específicos durante os 12 anos considerados no presente estudo.

As taxas de mortalidade por câncer de boca e faringe foram maiores nas regiões Sul e Sudeste do país, tanto nos resultados observados por Boing et al. ${ }^{6}$, quanto no presente trabalho. Sabe-se que o tabaco é o principal fator de risco para câncer de boca e faringe. As regiões Sul e Sudeste apresentam maior prevalência de consumo de tabaco do que as outras regiões ${ }^{24}$, o que pode estar afetando as taxas de mortalidade nessas regiões.

O estudo de tendências da mortalidade por câncer é complexo e sua análise deve considerar que as consequências potencialmente benéficas das intervenções em saúde só terão efeito em longo prazo, dada a ação cumulativa dos fatores de risco. Entre 1989 e 2010, a queda do percentual de fumantes no Brasil foi de $46 \%{ }^{16}$. Espera-se, então, que os seus efeitos estejam sendo refletidos na diminuição da mortalidade observada hoje, especialmente nas pessoas adultas que, durante anos, estiveram afetadas pelos efeitos da diminuição de consumo do tabaco.

Como possível consequência das políticas públicas destinadas ao controle do tabagismo e álcool, o país tem experimentado mudanças no consumo dessas substâncias, o que pode refletir nos diferentes padrões das tendências de mortalidade por câncer de boca e faringe. A diminuição observada no país na prevalência tabagismo ${ }^{16}$ pode influenciar a tendência em longo prazo das taxas de mortalidade por câncer de boca e faringe nos diferentes sítios anatômicos.

É importante salientar que, enquanto a incidência de câncer é passível de controle desde a prevenção primária, a mortalidade é susceptível à prevenção secundária por meio do diagnóstico oportuno e à prevenção terciária, que procura limitar o dano, controlar a dor, prevenir complicações secundárias e melhorar a qualidade de vida durante o tratamento ${ }^{25}$. Como não há evidência cientifica de que um exame visual, como parte de um programa de rastreamento de base populacional, reduza a taxa de mortalidade por câncer de boca e 
faringe, autores têm sugerido que estratégias focadas em indivíduos expostos aos principais fatores de risco poderiam resultar em uma prevenção secundária mais efetiva ${ }^{26}$.

Como todo estudo realizado com informações secundárias, os resultados aqui mostrados dependem da acurácia e completitude dos sistemas de informação. A qualidade e a abrangência dos dados de mortalidade fornecidos pelo SIM, têm aumentado gradativamente desde a sua descentralização em 1992 assim como o adequado preenchimento dos dados que se encontra em uma porcentagem ao redor de $90 \%{ }^{27}$. No Brasil há consistente avanço da cobertura desde o ano 2000 atingindo 96,1\% em 2011. Esta cobertura é próxima de 100\% nas regiões Sudeste, Sul e Centro-Oeste. Nas regiões Norte e Nordeste algumas unidades federativas têm cobertura acima de $90 \%$ e outras entre $80 \%$ e $90 \%$. O percentual de óbitos com causa básica mal definida apresenta queda ao longo dos anos, sendo as regiões Sul e Centro-Oeste aquelas com os menores percentuais de óbitos por causa básica mal definida (4,5\% e 4,4\%, respectivamente $)^{27}$. Segundo dados do Datasus ${ }^{\mathrm{e}}$, no período sob estudo, houve uma diminuição de mais de $80 \%$ no número de óbitos por causas mal definidas no país, sendo esta diminuição mais visível na região Nordeste.

As tendências observadas regionalmente podem estar sendo influenciadas pelas desigualdades regionais no registro dos dados sobre mortalidade e devem ser interpretadas com cautela, por exemplo, a redução da subnotificação de registros em regiões caracterizadas por baixa qualidade da assistência médica pode estar influenciando no aumento observado nas tendências. Nesse sentido, a tendência de crescimento refletiria antes uma melhoria de qualidade da informação do que um aumento na mortalidade.

Efeito análogo pode ser observado na diminuição das tendências das taxas do câncer de sítios não específicos de boca e faringe. A mortalidade por câncer de sítios específicos que não foram corretamente diagnosticados e registrados pode estar na verdade aumentando a proporção de óbitos por câncer de sítios não especificados no conjunto de registro de mortes. No período 2002-2013 as causas de morte classificadas como neoplasias de sítios não específicos de boca e faringe correspondentes aos códigos C06 e C14 da Classificação Internacional de Doenças, corresponderam a 30,6\% dos casos. A tendência de diminuição neste grupo específico pode estar sendo igualmente influenciada por melhorias na definição do sítio específico na causa de morte.

Além da qualidade dos dados obtidos nos sistemas de informação, os resultados estatísticos de uma análise de tendências são influenciados também pelo número de anos analisados. No presente estudo, ante uma série histórica relativamente curta, deve-se proceder com cautela nas interpretações das tendências.

Um aspecto importante é a capacidade de comparação dos resultados das taxas obtidas com estudos anteriores que usam outras populações para padronização. No entanto, o novo padrão usado foi adotado pela Organização Mundial da Saúde (OMS) visando a remover os efeitos da distribuição proporcional de diferentes populações no mundo, decorrente de acontecimentos históricos, guerras, fome etc. Isso converte esse novo padrão em uma população neutra para comparações e análises internacionais ${ }^{13}$.

Apesar das limitações próprias do estudo, nossos resultados mostram que as tendências estão ou estáveis, ou diminuindo. Boing et al. ${ }^{6}$ observaram aumento na mortalidade por câncer de orofaringe e hipofaringe, sítios anatômicos que, no presente estudo, apresentaram estabilidade e declínio, respectivamente. No citado estudo, sítios com tendência de diminuição, como lábios, língua, assoalho de boca e palato, passaram a mostrar estabilidade no período

e Ministério da Saúde (BR), Departamento de Informática do SUS - DATASUS. Informações de saúde: estatísticas vitais. Brasília (DF); c.2017 [citado 30 set 2017]. Disponível em: http://datasus.saude.gov.br/ informacoes-de-saude/tabnet/ estatisticas-vitais 2002-2013. A estabilização das taxas observada é o resultado de mudanças em longo prazo e indica melhorias no diagnóstico oportuno do câncer de boca e faringe e menor exposição aos fatores de risco. O comportamento diferenciado das tendências em cada macrorregião do país é reflexo das diferenças socioeconômicas, de acesso aos serviços de saúde e da melhoria dos sistemas de informação que impactam na variação de incidência, mortalidade e letalidade dessa doença. 


\section{CONCLUSÃO}

A mortalidade por câncer de faringe apresentou diminuição, enquanto câncer de boca apresentou estabilidade no período estudado. O câncer de boca apresentou tendência de aumento na região Nordeste e diminuição na região Sudeste, enquanto o câncer de faringe diminuiu nas regiões Sudeste, Sul e Centro-Oeste. As taxas de mortalidade por câncer de outras localizações mal definidas de lábio, cavidade oral e faringe mostraram declínio entre 2002 e 2013 no Brasil. Considerando o aumento observado na mortalidade por câncer de faringe no Brasil, observado no período 1979-2002, ressalta-se a diminuição observada no período 2002-2013 para a mortalidade por câncer nesse sítio anatômico e a estabilidade apresentada pelos demais sítios.

\section{REFERÊNCIAS}

1. Schramm JMA, Oliveira AF, Leite ID, Valente JG, Gadelha AMJ, Portela MC, et al. Transição epidemiológica e o estudo de carga de doença no Brasil. Cienc Saude Coletiva. 2004;9(4):897-908. https://doi.org/10.1590/S1413-81232004000400011

2. Garavello W, Bertuccio P, Levi F, Lucchini F, Bosetti C, Malvezzi M, et al. The oral cancer epidemic in central and eastern Europe. Int J Cancer. 2010;127(1):160-71. https://doi.org/10.1002/ijc.25019

3. Ariyawardana A, Johnson NW. Trends of lip, oral cavity and oropharyngeal cancers in Australia 1982-2008: overall good news but with rising rates in the oropharynx. BMC Cancer. 2013;13:333. https://doi.org/10.1186/1471-2407-13-333

4. Riera-S P, Martínez-R B. Morbilidad y mortalidad por cáncer oral y faríngeo en Chile. Rev Med Chile. 2005;133(5):555-63. https://doi.org/10.4067/S0034-98872005000500007

5. Wunsch Filho V. The epidemiology of oral and pharynx cancer in Brazil. Oral Oncol. 2002;38(8):737-46. https://doi.org/10.1016/S1368-8375(02)00066-0

6. Boing AF, Peres MA, Antunes JLF. Mortality from oral and pharyngeal cancer in Brazil: trends and regional patterns, 1979-2002. Rev Panam Salud Publica. 2006;20(1):1-8. https://doi.org/10.1590/S1020-49892006000700001

7. Weitkunat R, Sanders E, Lee PN. Meta-analysis of the relation between European and American smokeless tobacco and oral cancer. BMC Public Health. 2007;7:334. https://doi.org/10.1186/1471-2458-7-334

8. Turati F, Garavello W, Tramacere I, Pelucchi C, Galeone C, Bagnardi V, et al. A meta-analysis of alcohol drinking and oral and pharyngeal cancers: results from subgroup analyses. Alcohol Alcohol. 2013;48(1):107-18. https://doi.org/10.1093/alcalc/ags100

9. World health Organization. Global status report on alcohol and health 2014. Geneva: WHO; 2014 [citado 30 set 2017]. Disponível em: http://apps.who.int/iris/ bitstream/10665/112736/1/9789240692763_eng.pdf

10. Herrero R, Castellsagué X, Pawlita M, Lissowska J, Kee F, Balaram P, et al. Human papillomavirus and oral cancer: the International Agency for Research on Cancer multicenter study. I Natl Cancer Inst. 2003;95(23):1772-83. https://doi.org/10.1093/jnci/djg107

11. Bonafante GMS, Machado CJ, Souza PEA, Andrade EIG, Acurcio FA, Cherchiglia ML. Sobrevida de cinco anos e fatores associados ao câncer de boca para pacientes em tratamento oncológico ambulatorial pelo Sistema Único de Saúde, Brasil. Cad Saude Publica. 2014;30(5):983-97. https://doi.org/10.1590/0102-311X00182712

12. Klug C, Neuburg J,Glaser C, Schwarz B, Kermer C, Millesi W. Quality of life 2-10 years after combined treatment for advanced oral and oropharyngeal cancer. Int I Oral Maxillofac. Surg. 2002;31(6):664-9. https://doi.org/10.1054/ijom.2002.0301

13. Ahmad OB, Boschi-Pinto C, Lopez AD, Murray CJL, Lozano R, Inoue M. Age standardization of rates: a new WHO Standard. Geneva: World Heahl Organization; 2001 [citado 30 set 2017]. (GPE Discussion Paper Series, 31). Disponível em: http://www.who.int/healthinfo/paper31.pdf

14. Antunes JLF, Waldman EA. Trends and spatial distribution of deaths of children aged 12-60 months in São Paulo. Bull World Health Organ. 2002 [citado 30 set 2017];80(5):391-8. Disponível em: https://www.ncbi.nlm.nih.gov/pmc/articles/PMC2567796/pdf/12077615.pdf 
15. Yako-Suketomo H, Matsuda T. Comparison of time trends in lip, oral cavity and pharynx cancer mortality (1990-2006) between countries based on the WHO mortality database. Jpn J Clin Oncol. 2010;40(11):1118-9. https://doi.org/10.1093/jjco/hyq202

16. Biazevic MGH, Castellanos RA, Antunes JLF, Michel-Crosato E. Tendências de mortalidade por câncer de boca e orofaringe no Município de São Paulo, Brasil, 1980/2002. Cad Saude Publica. 2006;22(10):2105-14. https://doi.org/10.1590/S0102-311X2006001000016

17. Monteiro CA, Cavalcante TM, Moura EC, Claro RM, Szwarcwald CL. Population-based evidence of a strong decline in the prevalence of smokers in Brazil (1989-2003). Bull World Health Organ. 2007;85(7):527-34. https://doi.org/10.2471/BLT.06.039073

18. Machado LP, Camargo MBJ, Jeronymo JCM, Bastos GAN. Uso regular de serviços odontológicos entre adultos e idosos em região vulnerável no sul do Brasil. Rev Saude Publica. 2012;46(3):526-33. https://doi.org/10.1590/S0034-89102012000300015

19. Antunes JLF, Toporcov TN, Biazevic MGH, Boing AF, Bastos JL. Gender and racial inequalities in trends of oral cancer mortality in Sao Paulo, Brazil. Rev Saude Publica. 2013;47(3):470-8. https://doi.org/10.1590/S0034-8910.2013047003724

20. Oliveira JC, Curado MP, Martins E, Moreira MARM. Incidência, mortalidade e tendência do câncer de cavidade oral e orofaringe em Goiânia de 1988 a 2003. Rev Bras Cir Cabeça Pescoço. 2007 [citado 30 set 2017];36(2):70-4. Disponível em: http://www.sbccp.org.br/wp-content/ uploads/2014/11/2007_362-70-74. pdf

21. Secoli SR, Padilha KGP, Leite RCBO. Avanços tecnológicos em oncologia: reflexões para a prática de enfermagem. Rev Bras Cancerol. 2005 [citado 30 set 2017];51(4):331-7. Disponível em: http://www.inca.gov.br/rbc/n_51/v04/pdf/revisao4.pdf

22. Fajardo S, Aerts DRGC, Bassanesi SL. Acurácia da equipe do Sistema de Informações sobre Mortalidade na seleção da causa básica do óbito em capital no Sul do Brasil. Cad Saude Publica. 2009;25(10):2218-28. https://doi.org/10.1590/S0102-311X2009001000012

23. Rede Interagencial de Informação para a Saúde. Indicadores básicos para a saúde no Brasil: conceitos e aplicações. 2. ed. Brasília (DF): Organização Pan-Americana da Saúde; 2008 [citado 30 set 2017]. Disponível em: http://tabnet.datasus.gov.br/tabdata/livroidb/2ed/indicadores.pdf

24. Miranda CDC, Peres MA. Determinantes da utilização de serviços odontológicos entre adultos: um estudo de base populacional em Florianópolis, Santa Catarina. Cad Saude Publica. 2013;29(11):2319-32. https://doi.org/10.1590/0102-311X00139912

25. Instituto Brasileiro de Geografia e Estatística, Diretoria de Pesquisas, Coordenação de Trabalho e Rendimento. Pesquisa Nacional por Amostra de Domicílios: Tabagismo. Rio de Janeiro: IBGE; 2008 [citado 30 set 2017]. Disponível em: http://www1.inca.gov.br/inca/Arquivos/publicacoes/ tabagismo.pdf

26. Macpherson LMD, McCann MF, Gibson J, Binnie VI, Stephen KW. The role of primary healthcare professionals in oral cancer prevention and detection. Br Dent J. 2003;195(5):277-81. https://doi.org/10.1038/sj.bdj.4810481

27. Antunes JLF, Toporcov TN, Wunsch-Filho V. Resolutividade da campanha de prevenção e diagnóstico precoce do câncer bucal em São Paulo, Brasil. Rev Panam Salud Publica. 2007;21(1):30-6. https://doi.org/10.1590/S1020-49892007000100004

28. Ministério da Saúde (BR), Secretaria de Vigilância em Saúde, Coordenação Geral de Informações e Análises Epidemiológicas. Sistema de Informações sobre Mortalidade SIM: consolidação da base de dados de 2011. Brasília (DF); 2013 [citado 30 set 2017]. Disponível em: http://tabnet.datasus.gov.br/cgi/sim/Consolida_Sim_2011.pdf

Financiamento: Coordenação de Aperfeiçoamento do Pessoal de Nível Superior (CAPES).

Contribuição dos Autores: Concepção e planejamento do estudo: LMEP, MAP, AFB, JLFA. Coleta, análise e interpretação dos dados: LMEP, MAP, AFB, JLFA. Elaboração ou revisão do manuscrito: LMEP, MAP, AFB, JLFA. Aprovação da versão final: LMEP, MAP, AFB, JLFA. Responsabilidade pública pelo conteúdo do artigo: LMEP, MAP, AFB, JLFA.

Conflito de Interesses: Os autores declaram não haver conflito de interesses. 